\title{
Meta-Ribosomalomics: RNA Sequencing Is an Unbiased Method for Parasite Detection of Different Sample Types
}

\author{
Claudia Wylezich* and Dirk Höper \\ Friedrich-Loeffler-Institut, Institute of Diagnostic Virology, Greifswald-Insel Riems, Germany
}

In this perspective article, we review the past use of ribosomal sequences to address scientific and diagnostic questions. We highlight a variety of sequencing approaches including metagenomics and DNA barcoding and their different demands and requirements. Meta-ribosomalomics is introduced as an unbiased approach to exploit high-throughput sequencing datasets for eukaryotic and prokaryotic ribosomal sequences. Prerequisites, benefits, drawbacks, and future perspectives are elaborated and compared to other sequencing approaches.

\section{OPEN ACCESS}

Edited by: Magali Chabé,

Université de Lille, France

Reviewed by: Lucile Maria Floeter-Winter,

University of São Paulo, Brazil Satya P. Singh,

Saurashtra University, India

${ }^{*}$ Correspondence:

Claudia Wylezich

Claudia.Wylezich@ffl.de

Specialty section:

This article was submitted to Microbial Symbioses,

a section of the journal

Frontiers in Microbiology

Received: 06 October 2020

Accepted: 26 May 2021

Published: 21 June 2021

Citation:

Wylezich C and Höper D (2021)

Meta-Ribosomalomics: RNA

Sequencing Is an Unbiased Method for Parasite Detection of Different

Sample Types.

Front. Microbiol. 12:614553. doi: 10.3389/fmicb.2021.614553
Keywords: eukaryotic and prokaryotic diversity, parasites, ribosomal RNA, shotgun metagenomics, subtyping, taxonomic classification

\section{INTRODUCTION}

Sequencing of isolates and complex samples using extracted DNA has become a standard technique in microbiology and diagnostic laboratories. A major breakthrough in microbiology enabled the culture-independent characterization of microorganisms (i.e., single-celled eukaryotes and prokaryotes). An abundance of gene sequences was generated for either diversity surveys or diagnostic purposes. In microbial ecology, a PCR-based investigation of environmental samples was established, subsequently called meta-barcoding (amplicon-sequencing of short marker sequences). However, the application of PCR with the necessity of oligonucleotide primers introduces biases in the results of such analyses. The untargeted investigation of genomes of complex samples was alleviated by the introduction of new sequencing technologies and high-throughput sequencing (HTS). This approach, also known as metagenomics, can likewise be performed with extracted DNA (DNA-sequencing). Extracted RNA and subsequent depletion of ribosomal RNA (rRNA) is typically used to investigate the transcriptome of an organism or a complex sample, termed transcriptomics or meta-transcriptomics, respectively. In contrast, metagenomic analyses can also be performed on any RNA sample without depletion of the rRNA; in this case, the occurring ribosomal nucleic acids lead to sequencing datasets including reads of prokaryotic and eukaryotic rRNA. These reads comprise sequences of all rRNA molecules, i.e., the small subunit (SSU; comprising the historically defined $16 \mathrm{~S}$ and $18 \mathrm{~S}$ molecules) and large subunit (LSU; historically 23S, 28S) as well as internal transcribed spacer regions and 5S rRNA. As the majority of such RNA-based metagenomics datasets consists of ribosomal RNA, sequencing of the ribosomalome (all rRNA molecules of a complex sample) is dubbed meta-ribosomalomics. From our perspective, 
this approach has many benefits and advantages over primerdependent meta-barcoding and DNA-based metagenomics. As we present and discuss here, it can be an unbiased alternative to address diagnostic and ecological issues while allowing the parallel investigation of prokaryotic and eukaryotic diversity and the mutual interdependencies between both groups.

\section{SEQUENCING OF RIBOSOMAL RNA-FROM PAST TO FUTURE}

Sequencing of ribosomal RNA (rRNA) genes for phylogenetic purposes was in the focus for almost half a century. The small subunit (SSU) of rRNA genes of prokaryotes (16S rRNA gene) were intensely studied in the nineteen-seventies using oligonucleotide catalogs and first available sequencing methods (reviewed by Woese, 1987). About one decade later, small subunit of rRNA genes of single-celled eukaryotes (18S rRNA gene) were studied for the first time (see Figure $\mathbf{1}$ for details).

The development of the enzymatic polymerase chain reaction (PCR) in 1987 (Mullis and Faloona, 1987) revolutionized the repertoire of molecular methods and molecular diagnostics, and was also the starting point for the road to success for the characterization of ribosomal sequences and their use in molecular phylogeny. With the introduction of PCRs of complex environmental samples and their subsequent separation and amplification via bacterial cloning (clone libraries), plenty of complete and nearly complete SSU rRNA gene sequences became available and enabled addressing ecological research questions using the taxa composition derived from the sequence composition. The advent of new sequencing technologies and the application of HTS allowed massive parallel sequencing of environmental sequences circumventing their separation via bacterial cloning. However, in contrast to sequencing of isolates and environmental clones, only short fragments (variable regions, e.g., prokaryotic V3 or V6 and eukaryotic V4 or V9 SSU rDNA; Figure 1) instead of nearly complete genes were sequenced. Conversely, the usage of short sequences for phylogenetic reconstructions may be disadvantageous regarding the phylogenetic resolution of taxa (e.g., Choi and Park, 2020). A general drawback of the application of PCR for the analysis of prokaryotic and eukaryotic community composition is the primer bias (e.g., Geisen et al., 2015; Clarke et al., 2017 and references therein). This PCR bias in the analysis of environmental systems is based in part on incomplete sequencing of the rRNA molecules and in part on the inability of the universal primers to capture and hence amplify certain specific or lesser abundant molecules. Taken together, even potentially important taxa maybe discriminated by the use of universal primers. In addition, read numbers in sequencing datasets do not represent the initial cell abundance of a sample, as revealed by the inclusion of mock communities (Geisen et al., 2015; Wylezich et al., 2018a). This impedes the quantitative analysis of read numbers. Hence, the molecularly described diversity of a habitat depends on the choice of primers and gene region (Choi and Park, 2020) and is mostly a fragmentary picture. This distorts comparisons of results obtained from different studies using different primers.
Nevertheless, ribosomal sequences are the most used molecular markers to date. A search for prokaryotic and eukaryotic SSU rRNA gene sequences in GenBank (search criteria: ((((((rRNA) OR ribosomal RNA) AND 18S) OR 16S) OR SSU) NOT genome) NOT probe) on September 29, 2020, resulted in 9,269,968 entries. This is much more than for other often-used barcoding markers, for example cytochrome c (1,569,826 entries; search criteria: ((cytochrome c) NOT genome) NOT probe), or cytochrome oxidase 1 (2,799,587 entries; search criteria: (cytochrome oxidase 1) NOT genome) NOT probe). The above-mentioned number of SSU rRNA gene sequences includes many complete or nearly complete sequences. In addition to sequences in GenBank, plenty of short ribosomal sequence fragments of the variable barcoding regions (e.g., eukaryotic V4 and V9, prokaryotic V6) are deposited in short read archives $\left(S^{2} A^{1}\right)$ and even boost the number of available SSU rRNA reference sequences.

\section{CASE STUDIES OF META-RIBOSOMALOMICS FOR PARASITE DETECTION}

Diagnostic assays for the detection of pathogens including parasites typically target antigen (serologic detection and characterization) or nucleic acids (genetic detection and characterization); however, both approaches often lack standardization (Murray and Cappello, 2008). This is especially true for PCR assays for which different primer systems exist. Ribosomal sequences are used in clinical diagnostics from the early beginning and the reference database for ribosomal sequences of bacteria and parasites is huge. Meta-ribosomalomics can therefore be useful for parasite detection.

To overcome the limitations of the used primers, metaribosomalomics approaches hold a great promise. In a proofof-concept study, we re-analyzed metagenomics datasets of fecal samples of virus-infected pigs derived from total RNA and successfully extracted complete $18 \mathrm{~S}$ rRNA sequences of parasites (Wylezich et al., 2019a). Since the approach is PCRfree and does not rely on specific primers, it was possible to synchronously detect different parasites in one sample. Importantly, with this approach we could separate Blastocystis subtypes from each other and reported subtype 15 for the first time in pig feces. Hence, a hypothesis-free approach can be important to uncover the full diversity of species present in a sample which could be overlooked in case an expected species was detected and the analysis for other species stopped thereafter. In a follow-up study, the method was applied to fecal and organ samples pre-diagnosed for parasites (Wylezich et al., 2020). Diagnoses could be confirmed in nearly all cases. Further taxa (Dientamoeba, Iodamoeba, Endolimax, Hymenolepis) were synchronously recorded in addition to the pre-diagnosed ones. These results show the broad applicability of the approach and its discriminative power, which of course depends on the specificity of the in silico sequence analyses and the available

\footnotetext{
${ }^{1}$ https://www.ncbi.nlm.nih.gov/sra
} 
rRNA analyses through the ages

*community-based analyses

Examples for prokaryote studies

Examples for eukaryote / protistan studies

Sanger-sequencing of first ribosomal gene sequences

(e.g., oligonucleotide cataloging)
PCR-free HTS / meta-

ribosomalomics

Amplicon-based HTS / meta-barcoding *

Sanger-sequencing of clone libraries *

Sanger-sequencing of PCR-amplicons (isolates)

\begin{tabular}{r|c|c|}
\hline 1970 & 1980 \\
different 5S rRNA \\
(Fox \& Woese 1975) \\
16S rRNA E. coli \\
(Brosius et al. 1978) \\
18S rRNA of Dictyostelium dis- \\
coideum (McCarroll et al. 1983)
\end{tabular}

18S rRNA of Tetrahymena

thermophila (Spangler \& Blackburn 1985)

FIGURE 1 | Milestones of generating small-subunit ribosomal RNA gene sequences of prokaryotes and protistan eukaryotes to address phylogenetic and ecological questions (with references for the first application each; Fox and Woese, 1975; Brosius et al., 1978; McCarroll et al., 1983; Spangler and Blackburn, 1985; Medlin et al., 1988; Edwards et al., 1989; Giovannoni et al., 1990; Moon-van der Staay et al., 2001; Sogin et al., 2006; Amaral-Zettler et al., 2009; Wylezich et al., 2019a,b, 2020).

reference sequences. Noteworthy mentioning that the sensitivity also depends on the accessibility of the nucleic acids of the different pathogens, as stressed by Wylezich et al. (2018b).

Beside gut samples, organ samples of mice were screened for parasites using meta-ribosomalomics. The $18 \mathrm{~S}$ rRNA extracted from the resulting dataset allowed the molecular characterization of Klossiella cf. muris (available under accession numbers MT664760 and MT664769) using a complete 18S rRNA gene sequence for the first time. It exhibits a sequence identity of only $91.3 \%$ to the partial $18 \mathrm{~S}$ rRNA gene of $K$. equi (unpublished results).

Furthermore, we characterized a parasite in Malpighian tubules samples of bees via meta-ribosomalomics. This allowed us for the first time the phylogenetic characterization of the bee parasite Malpighamoeba mellificae based on 18S rRNA retrieved from a complex sequencing dataset (Wylezich et al., $2019 b)$. The actin gene could also be extracted from the dataset and supports the 18S rRNA phylogeny of $M$. mellificae as sister taxon of Micriamoeba tesseris within the class Tubulinea (Amoebozoa). Using 18S rRNA, a diagnostic PCR assay could be established for the first time for this often neglected bee parasite (unpublished results).

\section{RETRIEVING RIBOSOMAL RNA SEQUENCES FROM FIELD SAMPLES}

Meta-ribosomalomics is also applicable for field samples, for which typically amplicon-based 18S rRNA genes are used (e.g., Choi and Park, 2020). In proof-of-concept studies, we tested tap water samples (Wylezich et al., 2018b, and unpublished results) and groundwater samples (unpublished results). The datasets are currently being analyzed. First results indicate a high yield of $16 \mathrm{~S}$ and $18 \mathrm{~S}$ rRNA sequences that can be used to draw phylogenetic reconstructions and ecological considerations.

TABLE 1 | Overview of the prerequisites, current benefits and drawbacks of the meta-ribosomalomics approach.

\section{Prerequisites}

- RNA-friendly laboratory conditions are important to avoid RNA degradation

during sample processing

- Establishment and validation of the approach per laboratory

- Bioinformatics analysis needs an additional assembly step (in contrast to meta-barcoding)

\section{Benefits}

- Applicable for parallel detection of prokaryotes and eukaryotes in one sample

- No prior sequence information is necessary since it is an untargeted approach

- No primer bias exists since it is a PCR-free and primer-free approach

- Detection of unrecognized, unrelated taxa and co-infections is possible

- Resulting sequences can be a basis for designing better targeted approaches (e.g., for subsequent quantification purposes)

- Resulting datasets can be re-analyzed for protein-coding genes when more reference genomes of parasites are available

Drawbacks

- False-positives might be detected based on mis-assignments due to incorrectly curated genomes or erroneously named ribosomal sequences (database contaminations)

- High initial cost for establishment and validation of the approach per laboratory

Future perspective

- The possibility of quantification of detected taxa via found reads needs to be evaluated 
DNA-based metagenomics was used to investigate archaeal communities in a river estuary (Zou et al., 2020). Several genomes of ammonia-oxidizing Archaea were assembled from shotgun metagenomics datasets. $16 \mathrm{~S}$ and $23 \mathrm{~S}$ rDNA gene sequences retrieved from these assembled genomes, instead via PCR and amplicon-sequencing, were subsequently used for a phylogenetic characterization of the taxon.

In a direct comparison of SSU rDNA data from PCRgenerated metabarcoding with sequence fragments extracted from DNA-based shotgun metagenomics sequencing datasets, the latter provided more realistic estimates in terms of microbe community diversity and allowed a better representation of the rare biosphere taxa (Logares et al., 2014). This study points to the fact that a PCR step clearly biases the outcome of community analyses and can outcompete low-abundance taxa.

\section{DISCUSSION}

Easy to amplify ribosomal RNA genes (rDNA) are well known and have a long tradition as genetic markers for phylogenetic assignments. The ease of use gave rise to huge reference databases for ribosomal genes (e.g., SILVA ribosomal RNA database, Quast et al., 2013), accompanied by the development of numerous algorithms and software for the analysis of rRNA sequence data, for instance RNAmmer (Lagesen et al., 2007), Barrnap², SortMeRNA (Kopylova et al., 2012) or PhyloFlash (GruberVodicka et al., 2020). Ribosomal RNA (rRNA) is an abundant biomarker that can be used in clinical diagnostics in order to improve the detection limit of PCR-diagnostics in contrast to the use of rDNA (Hanron et al., 2017). This is due to the enormous abundance of rRNA transcripts in RNA-based datasets in contrast to rDNA in DNA-based datasets (Wylezich et al., 2019a and references therein). The meta-ribosomalomics approach takes advantage of the large amount of rRNA sequences in RNA-based datasets. Another advantage (see Table 1) is the complete absence of any primer bias since no target specific primers are applied to amplify target genes. Untargeted (PCR- and primer-free) approaches are well suited to catch hitherto unknown, unrelated or unexpected sequence types (Wylezich et al., 2019a,b, 2020).

In terms of abundance estimates and taxonomic classification, metagenomics whole-genome sequencing (based on DNA) shows a clearly better performance than meta-barcoding with rRNA amplicons as recently shown with mock communities (Logares et al., 2014; Khachatryan et al., 2020). However, the performance of RNA-based metagenomics (metaribosomalomics) regarding quantification purposes has yet to be investigated. Furthermore, the use of RNA for HTS has the advantage of drawing an immediate picture of the sample in contrast to DNA that can be derived from different sources including dead organisms or extracellular DNA (Pochon et al., 2017; Cristescu and Hebert, 2018).

In the above-mentioned examples, the generic metaribosomalomics approach was promising for diagnostics of

\footnotetext{
${ }^{2}$ https://github.com/tseemann/barrnap
}

typical but also neglected parasites and gut protists (e.g., Wylezich et al., 2019a,b, 2020). In addition, ribosomal gene sequences can be retrieved from DNA-based metagenomics datasets as shown for a novel species of ammonia-oxidizing Archaea (Zou et al., 2020). Regarding parasite characterization, missing well-curated reference genomes hamper correct read assignments and often deliver false-positive hits (e.g., Wylezich et al., 2019a). Therefore, ribosomal RNA gene sequences retrieved from metagenomics datasets (meta-ribosomalomics) might be more reliable to assign to the correct taxon than many protein-coding genes.

We mainly tested meta-ribosomalomics in the field of clinical diagnostics with a focus on eukaryotes. However, the attractiveness of the method is the parallel identification of prokaryotes and eukaryotes in a given sample via a single snapshot method. Therefore, the method is well suited to investigate gut microbiota with their prokaryotic and eukaryotic key players in one approach elucidating their complexity and interdependencies (Chabé et al., 2017). Thus, at least compositional trends of different gut microbiota can be explored using ribosomal genes. Based on such trends, candidate proteincoding genes can be subsequently retrieved from the datasets to prove and to deepen the initial findings. In addition, the method can provide the species and subtype inventory of microbiota based on ribosomal sequences together with information on viruses included in feces intended to fecal transplantations.

\section{AUTHOR CONTRIBUTIONS}

$\mathrm{CW}$ and $\mathrm{DH}$ conceived the study, wrote the manuscript, and approved it for publication. Both authors contributed to the article and approved the submitted version.

\section{FUNDING}

This work was supported by the EU funded project COMPARE (Grant Agreement No. 643476). CW was supported by the German Federal Ministry of Food and Agriculture through the Federal Office for Agriculture and Food (ZooSeq, Grant No. 2819114019).

\section{ACKNOWLEDGMENTS}

We would like to thank our collaborators, especially Marc O. Schäfer, Ulrich Schotte, and Rainer G. Ulrich, who were involved in unpublished studies, and the Topic Editors, especially Simone M. Cacciò, for the invitation to write this article. We thank Jacqueline King for amending the English text. We are grateful for the reviewers' comments that helped improve the manuscript. 


\section{REFERENCES}

Amaral-Zettler, L. A., McCliment, E. A., Ducklow, H. W., and Huse, S. M. (2009). A method for studying protistan diversity using massively parallel sequencing of V9 hypervariable regions of small-subunit ribosomal RNA genes. PLoS One 4:e6372. doi: 10.1371/journal.pone.0006372

Brosius, J., Palmer, M. L., Kennedy, J. P., and Noller, H. F. (1978). Complete nucleotide sequence of a $16 \mathrm{~S}$ ribosomal RNA gene from Escherichia coli. Proc. Natl. Acad. Sci. U.S.A. 75, 4801-4805.

Chabé, M., Lokmer, A., and Segurel, L. (2017). Gut protozoa: friends or foes of the human gut microbiota? Trends Parasitol. 33, 925-934. doi: 10.1016/j.pt.2017.08. 005

Choi, J., and Park, J. S. (2020). Comparative analyses of the V4 and V9 regions of $18 \mathrm{~S}$ rDNA for the extant eukaryotic community using the Illumina platform. Sci. Rep. 10:6519. doi: 10.1038/s41598-020-63561-z

Clarke, L. J., Beard, J. M., Swadling, K. M., and Deagle, B. E. (2017). Effect of marker choice and thermal cycling protocol on zooplankton DNA metabarcoding studies. Ecol. Evol. 7, 873-883. doi: 10.1002/ece3.2667

Cristescu, M. E., and Hebert, P. D. N. (2018). Uses and misuses of environmental DNA in biodiversity science and conservation. Annu. Ecol. Evol. Syst. 49, 209-230. doi: 10.1146/annurev-ecolsys-110617-062306

Edwards, U., Rogall, T., Blocker, H., Emde, M., and Bottger, E. C. (1989). Isolation and direct complete nucleotide determination of entire genes. Characterization of a gene coding for $16 \mathrm{~S}$ ribosomal RNA. Nucleic Acids Res. 17, 7843-7853. doi: $10.1093 /$ nar/17.19.7843

Fox, G. E., and Woese, C. R. (1975). 5S RNA secondary structure. Nature 256, 505-507. doi: 10.1038/256505a0

Geisen, S., Laros, I., Vizcaíno, A., Bonkowski, M., and de Groot, G. A. (2015). Not all are free-living: high-throughput DNA metabarcoding reveals a diverse community of protists parasitizing soil metazoa. Mol. Ecol. 24, 4556-4569. doi: $10.1111 /$ mec.13238

Giovannoni, S. J., Britschgi, T. B., Moyer, C. L., and Field, K. G. (1990). Genetic diversity in Sargasso Sea bacterioplankton. Nature 345, 60-63. doi: 10.1038/ 345060a0

Gruber-Vodicka, H. R., Seah, B. K. B., and Pruesse, E. (2020). phyloFlash: rapid small-subunit rRNA profiling and targeted assembly from metagenomes. mSystems 5:e00920-20. doi: 10.1128/mSystems.00920-20

Hanron, A. E., Billman, Z. P., Seilie, A. M., Chang, M., and Murphy, S. C. (2017). Detection of Babesia microti parasites by highly sensitive 18S rRNA reverse transcription PCR. Diagnost. Microbiol. Infect. Dis 87, 226-228. doi: 10.1016/ j.diagmicrobio.2016.11.021

Khachatryan, L., de Leeuw, R. H., Kraakman, M. E. M., Pappas, N., te Raa, M., Mei, H., et al. (2020). Taxonomic classification and abundance estimation using $16 \mathrm{~S}$ and WGS-a comparison using controlled reference samples. Forensic Sci. Int. Genet. 46:102257. doi: 10.1016/j.fsigen.2020.102257

Kopylova, E., Noé, L., and Touzet, H. (2012). SortMeRNA: fast and accurate filtering of ribosomal RNAs in metatranscriptomic data. Bioinformatics 28, 3211-3217. doi: 10.1093/bioinformatics/bts611

Lagesen, K., Hallin, P., Rødland, E. A., Staerfeldt, H. H., Rognes, T., and Ussery, D. W. (2007). RNAmmer: consistent and rapid annotation of ribosomal RNA genes. Nucleic Acids Res. 35, 3100-3108. doi: 10.1093/nar/gkm160

Logares, R., Sunagawa, S., Salazar, G., Cornejo-Castillo, F. M., Ferrera, I., Sarmento, H., et al. (2014). Using mitags to explore microbial communities. Environ. Microbiol. 16, 2659-2671. doi: 10.1111/1462-2920.12250

McCarroll, R., Olsen, G. J., Stahl, Y. D., Woese, C. R., and Sogin, M. L. (1983). Nucleotide sequence of the Dictyostelium discoideum small-subunit ribosomal ribonucleic acid inferred from the gene sequence: evolutionary implications. Biochemistry 22, 5858-5868.

Medlin, L. K., Elwood, H. J., Stickel, S., and Sogin, M. L. (1988). The characterization of enzymatically amplified eukaryotic 16S-like rRNA-coding regions. Gene 71, 491-499. doi: 10.1016/0378-1119(88)90066-2
Moon-van der Staay, S., De Wachter, R., and Vaulot, D. (2001). Oceanic 18S rDNA sequences from picoplankton reveal unsuspected eukaryotic diversity. Nature 409, 607-610. doi: 10.1038/35054541

Mullis, K. B., and Faloona, F. (1987). Specific synthesis of DNA in vitro via a polymerase-catalyzed chain reaction. Methods Enzymol. 155, 335-350. doi: 10. 1016/0076-6879(87)55023-6

Murray, T. S., and Cappello, M. (2008). The molecular diagnosis of parasitic diseases. Pediatr. Infect. Dis. J. 27, 163-164.

Pochon, X., Zaiko, A., Fletcher, L. M., Laroche, O., and Wood, S. A. (2017). Wanted dead or alive? Using metabarcoding of environmental DNA and RNA to distinguish living assemblages for biosecurity applications. PLoS One 12:e0187636. doi: 10.1371/journal.pone.018 7636

Quast, C., Pruesse, E., Yilmaz, P., Gerken, J., Schweer, T., Yarza, P., et al. (2013). The SILVA ribosomal RNA gene database project: improved data processing and web-based tools. Nucleic Acids Res. 41, D590-D596. doi: 10.1093/nar/gks 1219

Sogin, M. L., Morrison, H. G., Huber, J. A., Welch, D. M., Huse, S. M., Neal, P. R., et al. (2006). Microbial diversity in the deep sea and the underexplored, "rare biosphere". Proc. Nat. Acad. Sci. U.S.A. 103, 12115-12120. doi: 10.1073/pnas. 0605127103

Spangler, E. A., and Blackburn, E. H. (1985). The nucleotide sequence of the $17 \mathrm{~S}$ ribosomal RNA gene of Tetrahymena thermophila and the identification of point mutations resulting in resistance to the antibiotics paromomycin and hygromycin. J. Biol. Chem. 260, 6334-6340.

Woese, C. R. (1987). Bacterial evolution. Microbiol. Rev. 51, 221-271.

Wylezich, C., Belka, A., Hanke, D., Beer, M., Blome, S., and Höper, D. (2019a). Metagenomics for broad and improved parasite detection: a proof-of-concept study using swine faecal samples. Int. J. Parasitol. 49, 769-777. doi: 10.1016/j. ijpara.2019.04.007

Wylezich, C., Caccio, S. M., Walochnik, J., Beer, M., and Höper, D. (2020). Untargeted metagenomics shows a reliable performance for synchronous detection of parasites. Parasitol. Res. 119, 2623-2629. doi: 10.1007/s00436-02006754-9

Wylezich, C., Herlemann, D. P. R., and Jürgens, K. (2018a). Improved 18S rDNA amplification protocol for assessing protist diversity in oxygendeficient marine systems. Aquat. Microb. Ecol. 81, 83-94. doi: 10.3354/ame 01864

Wylezich, C., Höper, D., Beer, M., and Schäfer, M. O. (2019b). "First phylogenetic characterization of Malpighamoeba mellificae using gene sequences extracted from a metagenomic deep sequencing approach reveals affiliation to the Tubulinea," in Proceedings of the Annual Meeting of the German Society for Protistology, (Vienna).

Wylezich, C., Papa, A., Beer, M., and Höper, D. (2018b). A versatile sample processing workflow for diagnostic metagenomics. Sci. Rep. 8:13108. doi: 10 . 1038/s41598-018-31496-1

Zou, D., Wan, R., Han, L., Xu, M. N., Liu, Y., Liu, H., et al. (2020). Genomic characteristics of a novel species of ammonia-oxidizing archaea from the Jiulong River estuary. Appl. Environ. Microbiol. 86:e00736-20. doi: 10.1128/ AEM.00736-20

Conflict of Interest: The authors declare that the research was conducted in the absence of any commercial or financial relationships that could be construed as a potential conflict of interest.

Copyright (C) 2021 Wylezich and Höper. This is an open-access article distributed under the terms of the Creative Commons Attribution License (CC BY). The use, distribution or reproduction in other forums is permitted, provided the original author(s) and the copyright owner(s) are credited and that the original publication in this journal is cited, in accordance with accepted academic practice. No use, distribution or reproduction is permitted which does not comply with these terms. 